J Stat Phys manuscript No.

(will be inserted by the editor)

Ladislav Šamaj · Emmanuel Trizac

\title{
Counter-ions between or at asymmetrically charged walls: 2D free-fermion point
}

Received: / Accepted:

\begin{abstract}
This work contributes to the problem of determining effective interaction between asymmetrically (likely or oppositely) charged objects whose total charge is neutralized by mobile pointlike counter-ions of the same charge, the whole system being in thermal equilibrium. The problem is formulated in two spatial dimensions with logarithmic Coulomb interactions. The charged objects correspond to two parallel lines at distance $d$, with fixed line charge densities. Two versions of the model are considered: the standard "unconstrained" one with particles moving freely between the lines and the "constrained" one with particles confined to the lines. We solve exactly both systems at the free-fermion coupling and compare the results for the pressure (i.e. the force between the lines per unit length of one of the lines) with the mean-field Poisson-Boltzmann solution. For the unconstrained model, the large- $d$ asymptotic behaviour of the free-fermion pressure differs from that predicted by the mean-field theory. For the constrained model, the asymptotic pressure coincides with the attractive van der Waals-Casimir fluctuational force. For both models, there are fundamental differences between the cases of likely-charged and oppositely-charged lines, the latter case corresponding at large distances $d$ to a capacitor.
\end{abstract}

Keywords Logarithmic Coulomb interaction · Free-fermion point · Exactly solvable models · van der Waals-Casimir force

Ladislav Šamaj · Emmanuel Trizac

Université Paris-Sud, Laboratoire de Physique Théorique et Modèles Statistiques, UMR CNRS 8626, 91405 Orsay, France

Ladislav Šamaj

Institute of Physics, Slovak Academy of Sciences, Dúbravská cesta 9, 84511

Bratislava, Slovakia 


\section{Introduction}

We study classical (i.e. non-quantum) systems of particles interacting pairwisely by the Coulomb potential, which are in thermal equilibrium. Such systems are of practical interest in the "real" three-dimensional (3D) world where the Coulomb potential has the standard $1 / r$ form. One can extend its definition to any dimension $\nu=1,2, \ldots$ in the following way: the Coulomb potential $v$ at a spatial position $\mathbf{r} \in \mathbb{R}^{\nu}$, induced by a unit charge at the origin $\mathbf{0}$, is the solution of the Poisson equation

$$
\Delta v(\mathbf{r})=-s_{\nu} \delta(\mathbf{r}),
$$

where $s_{\nu}=2 \pi^{\nu / 2} / \Gamma(\nu / 2)[\Gamma(x)$ denotes the Gamma function] is the surface area of the $\nu$-dimensional unit sphere. In particular,

$$
v(\mathbf{r})= \begin{cases}-\ln \left(r / r_{0}\right) & \text { if } \nu=2, \\ \frac{r^{2-\nu}}{\nu-2} & \text { otherwise }\end{cases}
$$

where $r \equiv|\mathbf{r}|$ and an arbitrary length scale $r_{0}$ fixes the zero of the twodimensional (2D) Coulomb potential. Such definition implies in the Fourier space the characteristic $1 / k^{2}$ behaviour which maintains many generic properties of 3D Coulomb systems like screening [17]. This is why many important phenomena related to the Coulomb law manifest themselves in a similar way in various spatial dimensions.

2D one-component (jellium) and symmetric two-component (Coulomb gas) systems with logarithmic charge interactions are of special importance because they are exactly solvable, besides the mean-field weak-coupling limit, also at a specific finite temperature. The solvable cases involve the bulk regime [11,7] as well as inhomogeneous, semi-infinite or fully finite geometries [8, see reviews [12,9]. This permits us to check basic concepts or ideas on $2 \mathrm{D}$ exact solutions.

One of extensively studied problems in our days is the electromagnetic Casimir effect, see e.g. 2 and 18. In its formulation within classical statistical mechanics, two conducting neutral slabs in thermal equilibrium [at the inverse temperature $\beta=1 /\left(k_{\mathrm{B}} T\right)$ ], being from one another at distance $d$ much larger than any microscopic (e.g. Debye-Hückel) length scale, are attracted by the long-range van der Waals-Casimir force. This force is due to thermal fluctuations of local charge densities inside globally neutral conductors. In $\nu$ spatial dimensions, the force per unit area of one of the slabs, or equivalently the pressure $P$, was obtained in the form [13]

$$
\beta P=-\frac{(\nu-1) \zeta(\nu) \Gamma(\nu / 2)}{2^{\nu} \pi^{\nu / 2}} \frac{1}{d^{\nu}} \quad(\text { dimension } \nu),
$$

where $\zeta(\nu)=\sum_{n=1}^{\infty} n^{-\nu}$ is the Riemann zeta function and the minus/plus sign means attraction/repulsion of the slabs. Note that in one dimension, one 
gets a finite result owing to the limiting behaviour $\lim _{\nu \rightarrow 1}(\nu-1) \zeta(\nu)=1$. In $2 \mathrm{D}$, we have explicitly

$$
\beta P=-\frac{\pi}{24} \frac{1}{d^{2}} \quad(2 \mathrm{D}),
$$

and in 3D

$$
\beta P=-\frac{\zeta(3)}{8 \pi} \frac{1}{d^{3}} \quad(3 \mathrm{D}) .
$$

These large-distance behaviours do not depend on the composition of conducting slabs, nor on the temperature: they are universal. It was shown 3 . that the microscopic origin of this universality lies in an electroneutrality sum rule for Coulomb fluids.

Another basic problem in soft matter physics is the determination of the temperature-dependent effective interaction between charged mesoscopic objects, macro-ions (colloids, polyelectrolytes) or charged membranes of several thousands elementary charges $e$, immersed in a polar solvent, e.g. water, containing mobile micro-ions of low valence. Let the large macro-ions be approximated by rectilinear hard walls, filled by a material of the same dielectric constant as the solvent (i.e. there are no electrostatic image charges). In a simplified model coined as "counter-ions only", or "salt-free", the macroions acquire a fixed surface charge density $\sigma e(\sigma>0)$ by releasing equally charged $-e$ (valence $=1$, for simplicity) counter-ions into the solvent, the system as a whole being electroneutral. This model was studied in the semiinfinite geometry of one charged wall and for counter-ions being in between two symmetrically charged parallel plates at distance $d$. It became the cornerstone for studying systematically the weak-coupling (high-temperature) 19 and strong-coupling (low-temperature) 20,24 limit of Coulomb fluids. While two symmetrically charged plates always repel one another in the weak-coupling limit, the situation changes for sufficiently large couplings: an attraction may occur in a certain interval of distances $d$. An important information in the two-plate geometry is the asymptotic $d \rightarrow \infty$ behaviour of the pressure, namely its sign (attraction or repulsion) and the explicit inverse-power-law dependence on $d$. Naively, one may expect that for large $d$, the counter-ions will separate onto two sets, one for each plate, to neutralize these plates. Then each plate with its counter-ions might be viewed as a "neutral slab" and the two slabs interact consequently by the attractive van der Waals-Casimir force of type (3). But the Poisson-Boltzmann solution [1, generalized straightforwardly from $3 \mathrm{D}$ to any dimension $\nu$,

$$
\beta P \underset{\sigma d \rightarrow \infty}{\sim} \frac{2 \pi^{2}}{\beta e^{2} s_{\nu}} \frac{1}{d^{2}} \quad(\text { dimension } \nu)
$$

shows that the possibility of counter-ions to migrate in the space between the plates is crucial. This force is repulsive and differs fundamentally from the attractive fluctuational one (3): although the prefactor to $1 / d^{2}$ is independent of the fixed surface charge density $\sigma e$, it does depend on the temperature. An important question is whether the mean-field asymptotic behaviour (6) remains valid also for finite temperatures. Such supposition is often taken as natural, without any rigorous proof. 
For the related geometry of one wall, the density profiles of counter-ions were investigated within a test-charge theory for the electric double layer in ref. 4. This theory provides results which interpolate between the correct strong-coupling (exponential decay) and the weak-coupling (algebraic decay) limits. Its application to intermediate values of the coupling constant indicates that there is a crossover from exponential to algebraic decay at large distance from the charged wall. It is argued that in the algebraic regime, the density profile is described by a modified mean-field equation. This unexpected conclusion might be put into doubts by the fact that the exact contact theorem for the particle density at the wall is not fulfilled by the test-charge theory in the region of intermediate values of the coupling constant.

For symmetrically charged lines in $2 \mathrm{D}$, the mean-field formula for the pressure (6) takes the form

$$
\beta P \underset{\sigma d \rightarrow \infty}{\sim} \frac{\pi}{\Gamma} \frac{1}{d^{2}} \quad(\Gamma \rightarrow 0)
$$

where $\Gamma \equiv \beta e^{2}$ is the $2 \mathrm{D}$ coupling constant. In a recent work 25 , we solved exactly the free-fermion point $\Gamma=2$ by using a technique of anticommuting variables, with the result

$$
\beta P=\frac{1}{\pi d^{2}} \int_{0}^{2 \pi \sigma d} \mathrm{~d} s \frac{s}{\sinh s} \mathrm{e}^{-s} \underset{\sigma d \rightarrow \infty}{\sim} \frac{\pi}{12} \frac{1}{d^{2}} \quad(\Gamma=2) .
$$

This asymptotic behaviour is not compatible with the mean-field one (7), i.e. the mean-field prefactor must be renormalized by a $\Gamma$-dependent function which goes to unity as $\Gamma \rightarrow 0$.

The aim of this paper is twofold.

- First, for the "unconstrained" version of the model with counter-ions moving freely in space between the lines, we generalize the exact result at $\Gamma=2$ for symmetric lines 25] to asymmetrically charged lines, see Fig. 1. The weak-coupling and strong-coupling limits for 3D asymmetrically charged plates were investigated in ref. 14 . The $2 \mathrm{D}$ exact result at the finite free-fermion coupling $\Gamma=2$, presented in this work, leads to a pressure which consists of two decoupled contributions from each of the lines.

- Our second aim is to solve a "constrained" version of the model in which counter-ions do not move freely between the asymmetrically charged lines, but are constrained to them (Fig. 2). The particle occupation of the lines is not fixed, i.e. the particles can lie either on $x=0$ line or $x=d$ line. A similar 3D model, in which charged particles are constrained to and simultaneously neutralize each of the symmetrically-charged plates, was introduced and studied at low temperatures in refs. 15. 16. We show that our constrained model yields a phenomenology that is close to the Casimir electromagnetic effect, although, at each finite distance, the net charge of every line is non-zero and thus the charged lines together with the attached particles do not describe neutral conductors as required by the Casimir effect. 
For both models, there are important differences between the cases of likelycharged and oppositely-charged lines, the latter case corresponds at large distances to a capacitor.

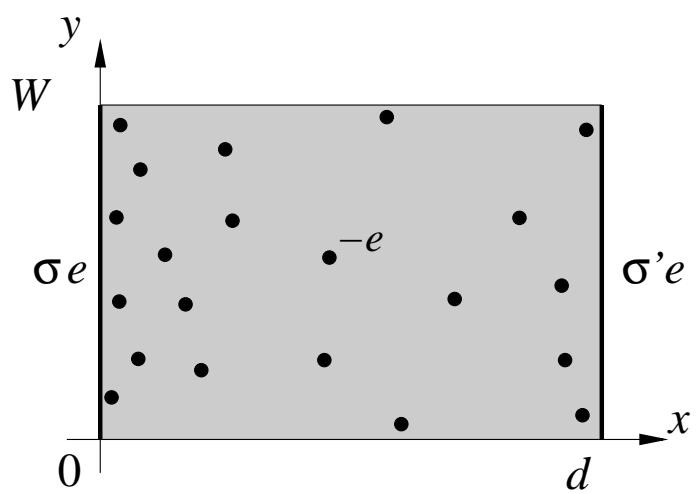

Fig. 1 Cylinder geometry with periodical boundary conditions (period $W$ ) along the $y$-axis. Two parallel lines with fixed charge densities $\sigma e$ and $\sigma^{\prime} e$ are localized at the end points $x=0$ and $x=d$, respectively. Pointlike counter-ions of charge $-e$ are allowed to move freely between the lines in the "unconstrained" version of the model.

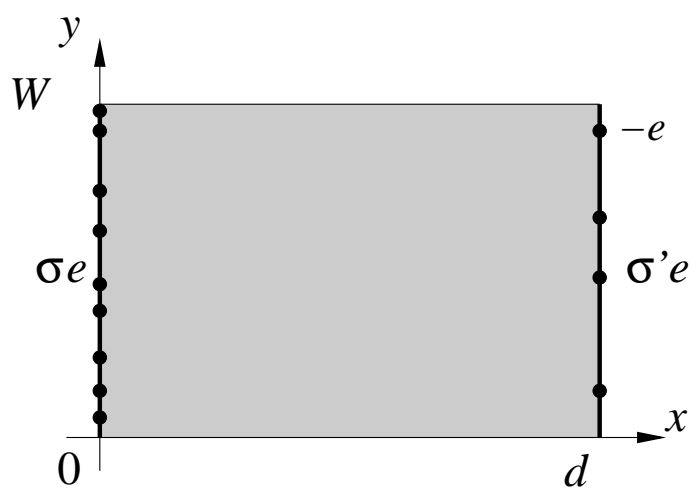

Fig. 2 "Constrained" version of the model: Cylinder geometry with asymmetrically charged lines, where counter-ions' displacements are limited to the lines.

The paper is organized as follows. In Sect. 2, we recapitulate briefly the formalism of anticommuting variables for a cylinder geometry of the confining domain, with two asymmetrically charged circles at distance $d$. The thermodynamic limit is reached by increasing the cylinder circumference $W$ to infinity, keeping the overall electroneutrality, which effectively transforms discrete sums to continuous integrals. Sect. 3 is devoted to the solution of 
the unconstrained model. In Sect. 4, we deal with the model of counter-ions constrained to the charged lines for which a comparison is made for large distances $d$ with the van der Waals-Casimir asymptotic formula (44). The qualitatively distinct regimes with likely-charged and oppositely-charged lines are analyzed for both models. A short summary and conclusions are drawn in Sect. [5.

\section{General formalism for cylinder geometry}

We consider the system of $N$ mobile pointlike particles with the (minus) elementary charge $-e$, confined to the surface of a cylinder of circumference $W$ and finite length $d$. The surface of the cylinder can be represented as a $2 \mathrm{D}$ semiperiodic rectangle domain $\Lambda$ of points $\mathbf{r}=(x, y)$ with coordinates $x \in[0, d]$ (free boundary conditions at $x=0, d$ ) and $y \in[0, W]$ (periodic boundary conditions at $y=0, W$ ). It is useful to introduce the complex coordinates $z=x+\mathrm{i} y$ and $\bar{z}=x-\mathrm{i} y$. There are fixed line charge densities $\sigma e$ and $\sigma^{\prime} e$ of dimension [length $]^{-1}$ along the $y$-axis at the end-points $x=0$ and $x=d$, respectively. Without any loss of generality, we restrict ourselves to

$$
\sigma>0, \quad-\sigma<\sigma^{\prime} \leq \sigma,
$$

which therefore includes cases where $\sigma^{\prime}<0$. We introduce the asymmetry parameter

$$
\eta \equiv \frac{\sigma^{\prime}}{\sigma}, \quad \eta \in(-1,1]
$$

The symmetric case corresponds to $\eta=1$. The overall electroneutrality condition is expressed as

$$
N=\left(\sigma+\sigma^{\prime}\right) W .
$$

Note that the requirement of positivity of $N$ corresponds to the restriction $-\sigma<\sigma^{\prime}$ in (9). We are interested in the thermodynamic limit $N, W \rightarrow \infty$, keeping the ratio $N / W=\sigma+\sigma^{\prime}$ fixed.

The Coulomb potential $v$ at a spatial position $\mathbf{r} \in \Lambda$, induced by a unit charge at the origin $\mathbf{0}$, is defined as the solution of the $2 \mathrm{D}$ Poisson equation $\Delta v(\mathbf{r})=-2 \pi \delta(\mathbf{r})$, under the periodicity requirement along the $y$-axis with period $W$. Considering the potential as a Fourier series in $y$, one obtains [6]

$$
\begin{aligned}
v(\mathbf{r}) & =-\ln \left|2 \sinh \left(\frac{\pi z}{W}\right)\right| \\
& =-\frac{1}{2} \ln \left[2 \cosh \left(\frac{2 \pi x}{W}\right)-2 \cos \left(\frac{2 \pi y}{W}\right)\right] .
\end{aligned}
$$

For small distances $\mathbf{r} \ll W$, this potential reduces to the $2 \mathrm{D}$ Coulomb potential $-\ln (2 \pi r / W)$. At large distances along the cylinder $x \gg W$, this potential behaves like the $1 \mathrm{D}$ Coulomb potential $-\pi|x| / W$. The interaction potential of two unit charges at points $\mathbf{r}$ and $\mathbf{r}^{\prime}$ is given by $v\left(\mathbf{r}, \mathbf{r}^{\prime}\right)=v\left(\left|\mathbf{r}-\mathbf{r}^{\prime}\right|\right)$. In what follows, we shall use two formulas:

$$
\int_{0}^{W} \mathrm{~d} y v(\mathbf{r})=-\pi x
$$


and

$$
\left|2 \sinh \frac{\pi\left(z-z^{\prime}\right)}{W}\right|=\mathrm{e}^{\frac{\pi}{W}\left(x+x^{\prime}\right)}\left|\mathrm{e}^{-\frac{2 \pi}{W} z}-\mathrm{e}^{-\frac{2 \pi}{W} z^{\prime}}\right| .
$$

For a given spatial configuration $\left\{\mathbf{r}_{1}, \cdots, \mathbf{r}_{N}\right\}$ of $N$ charges, the total Coulomb energy of the system consists of the self and mutual interactions of the fixed surface charge densities $\sigma e$ and $\sigma^{\prime} e, E_{s s}$, particles and surface charge densities, $E_{p s}$, and particles themselves, $E_{p p}$. Using the relation (13) and the notation $v(\mathbf{r}) \equiv v(x, y)$, the self-interaction of the surface charge densities $\sigma e$ and $\sigma^{\prime} e$ vanishes,

$$
\frac{1}{2}\left[(\sigma e)^{2}+\left(\sigma^{\prime} e\right)^{2}\right] \int_{0}^{W} \mathrm{~d} y \int_{0}^{W} \mathrm{~d} y^{\prime} v\left(0, y-y^{\prime}\right)=0,
$$

while their mutual interaction energy is given by

$$
(\sigma e)\left(\sigma^{\prime} e\right) \int_{0}^{W} \mathrm{~d} y \int_{0}^{W} \mathrm{~d} y^{\prime} v\left(d, y-y^{\prime}\right)=-\pi(\sigma e)\left(\sigma^{\prime} e\right) W d
$$

Since $(-e)(\sigma e) \int_{0}^{W} \mathrm{~d} y v(x, y)=\pi \sigma e^{2} x$, the interaction energy of particles with the surface charge densities takes the form

$$
\begin{aligned}
E_{p s} & =\sum_{j=1}^{N}\left[\pi \sigma e^{2} x_{j}+\pi \sigma^{\prime} e^{2}\left(d-x_{j}\right)\right] \\
& =\sum_{j=1}^{N} \pi\left(\sigma-\sigma^{\prime}\right) e^{2} x_{j}+N \pi \sigma^{\prime} e^{2} d .
\end{aligned}
$$

The particle-particle interaction energy is simply given by

$$
E_{p p}=\sum_{(j<k)=1}^{N} e^{2} v\left(\left|\mathbf{r}_{j}-\mathbf{r}_{k}\right|\right)
$$

At inverse temperature $\beta=1 /\left(k_{\mathrm{B}} T\right)$, the Boltzmann factor of the total energy $E_{N}(\{\mathbf{r}\})=E_{s s}+E_{p s}+E_{p p}$ reads

$$
\begin{aligned}
\mathrm{e}^{-\beta E_{N}(\{\mathbf{r}\})=} & \mathrm{e}^{-\pi \Gamma\left(\sigma^{\prime}\right)^{2} W d} \prod_{j=1}^{N} \mathrm{e}^{\pi \Gamma\left(\sigma^{\prime}-\sigma\right) x_{j}} \\
& \times \prod_{(j<k)=1}^{N}\left|2 \sinh \frac{\pi\left(z_{j}-z_{k}\right)}{W}\right|^{\Gamma},
\end{aligned}
$$

where $\Gamma=\beta e^{2}$ is the coupling constant.

Within the canonical ensemble, the partition function is defined as

$$
Z_{N}=\frac{1}{N !} \int_{\Lambda} \frac{\mathrm{d} \mathbf{r}_{1}}{\lambda^{2}} \epsilon\left(\mathbf{r}_{1}\right) \cdots \int_{\Lambda} \frac{\mathrm{d} \mathbf{r}_{N}}{\lambda^{2}} \epsilon\left(\mathbf{r}_{N}\right) \mathrm{e}^{-\beta E_{N}(\{\mathbf{r}\})},
$$


where $\lambda$ is the thermal de Broglie wavelength and the function $\epsilon(\mathbf{r})$ reflects a possible constraint to the location of particles. In particular,

$$
\epsilon(\mathbf{r})=1
$$

for the unconstrained model pictured in Fig. 1 1 and

$$
\epsilon(\mathbf{r}) \equiv \epsilon(x)=\lambda[\delta(x)+\delta(x-d)]
$$

for the constrained model in Fig. 2. Here, $\lambda$ is included for dimensional reason, and turns immaterial in what follows. The introduction of the $\epsilon$-function allows us to treat the two models using the same technique. With the aid of formula (14), the partition function (20) can be expressed as

$$
Z_{N}=\left(\frac{W^{2}}{4 \pi \lambda^{2}}\right)^{N} \exp \left[-\pi \Gamma\left(\sigma^{\prime}\right)^{2} W d\right] Q_{N}
$$

where

$$
Q_{N}=\frac{1}{N !} \int_{\Lambda} \prod_{j=1}^{N}\left[\mathrm{~d}^{2} z_{j} w_{\mathrm{ren}}\left(x_{j}\right)\right] \prod_{j<k}\left|\mathrm{e}^{-\frac{2 \pi}{W} z_{j}}-\mathrm{e}^{-\frac{2 \pi}{W} z_{j}}\right|^{\Gamma}
$$

and

$$
\begin{aligned}
w_{\text {ren }}(x) & =\frac{4 \pi}{W^{2}} \epsilon(x) \exp \left[\pi \Gamma\left(\sigma^{\prime}-\sigma\right) x+\frac{\pi \Gamma}{W}(N-1) x\right] \\
& =\frac{4 \pi}{W^{2}} \epsilon(x) \exp \left[\pi \Gamma\left(2 \sigma^{\prime}-\frac{1}{W}\right) x\right]
\end{aligned}
$$

is the renormalized one-body Boltzmann factor.

For $\Gamma=2 \gamma$ ( $\gamma$ a positive integer $)$, the technique of anticommuting variables [25,22,23 allows us to express $Q_{N}$ as the integral over Grassman variables with the action that couples certain composite operators by interaction strengths

$$
w_{j k}=\int_{\Lambda} \mathrm{d}^{2} z w_{\text {ren }}(x) \exp \left(-\frac{2 \pi}{W} j z\right) \exp \left(-\frac{2 \pi}{W} k \bar{z}\right)
$$

$[j, k=0,1, \ldots, \gamma(N-1)]$. Due to the orthogonality relation

$$
\int_{0}^{W} \mathrm{~d} y \exp \left[\frac{2 \pi}{W} \mathrm{i}(k-j) y\right]=W \delta_{j k},
$$

the interaction matrix becomes diagonal, $w_{j k}=w_{j} \delta_{j k}$ with

$$
w_{j}=W \int_{0}^{d} \mathrm{~d} x w_{\text {ren }}(x) \exp \left(-\frac{4 \pi}{W} j x\right)
$$

$[j=0,1, \ldots, \gamma(N-1)]$. 
At the special coupling $\Gamma=2(\gamma=1)$, the composite operators become the standard anticommuting variables. Due to the diagonalized form of the action

$$
S=\sum_{j=0}^{N-1} \xi_{j} w_{j} \psi_{j}
$$

we obtain the exact result

$$
Q_{N}=\prod_{j=0}^{N-1} w_{j} .
$$

Omitting in (23) the irrelevant $d$-independent prefactors, the free energy, defined by $-\beta F_{N}=\ln Z_{N}$, reads

$$
-\beta F_{N}=-2 \pi\left(\sigma^{\prime}\right)^{2} W d+\sum_{j=0}^{N-1} \ln w_{j} .
$$

The corresponding pressure $P_{N}$, i.e. the force between the plates per unit length of one of the plates, is given by

$$
\beta P_{N}=\frac{\partial}{\partial d}\left(\frac{-\beta F_{N}}{W}\right) .
$$

Explicitly,

$$
\beta P_{N}=-2 \pi\left(\sigma^{\prime}\right)^{2}+\frac{1}{W} \sum_{j=0}^{N-1} \frac{1}{w_{j}} \frac{\partial w_{j}}{\partial d} .
$$

Moreover, using the formalism of anticommuting variables, the density profile of particles is expressible as

$$
n(x)=w_{\text {ren }}(x) \sum_{j=0}^{N-1}\left\langle\xi_{j} \psi_{j}\right\rangle \exp \left(-\frac{4 \pi}{W} j x\right),
$$

where the correlators are given by $\left\langle\xi_{j} \psi_{j}\right\rangle=1 / w_{j}(j=0, \ldots, N-1)$ within the free-fermion action (29).

\section{Counter-ions between charged walls}

We first consider the unconstrained model in Fig. 1 1 with the trivial $\epsilon$-function given by (21). At $\Gamma=2$, the renormalized one-body weight (25) reads

$$
w_{\text {ren }}(x)=\frac{4 \pi}{W^{2}} \exp \left[2 \pi\left(2 \sigma^{\prime}-\frac{1}{W}\right) x\right]
$$

and the diagonal interaction elements (28) are given by

$$
w_{j}=\frac{1}{j-W \sigma^{\prime}+\frac{1}{2}}\left[1-\mathrm{e}^{-\frac{4 \pi d}{W}\left(j-W \sigma^{\prime}+\frac{1}{2}\right)}\right]
$$


$(j=0,1, \ldots, N-1)$. The pressure (33) is then expressible as

$$
\begin{aligned}
\beta P_{N}= & -2 \pi\left(\sigma^{\prime}\right)^{2}+\frac{4 \pi}{W^{2}} \sum_{j=0}^{N-1} \frac{j-W \sigma^{\prime}+\frac{1}{2}}{1-\mathrm{e}^{-\frac{4 \pi d}{W}\left(j-W \sigma^{\prime}+\frac{1}{2}\right)}} \\
& \times \mathrm{e}^{-\frac{4 \pi d}{W}\left(j-W \sigma^{\prime}+\frac{1}{2}\right)}
\end{aligned}
$$

and the density profile (34) as

$$
n(x)=\frac{4 \pi}{W^{2}} \sum_{j=0}^{N-1} \frac{j-W \sigma^{\prime}+\frac{1}{2}}{1-\mathrm{e}^{-\frac{4 \pi d}{W}\left(j-W \sigma^{\prime}+\frac{1}{2}\right)}} \mathrm{e}^{-\frac{4 \pi x}{W}\left(j-W \sigma^{\prime}+\frac{1}{2}\right)} .
$$

The particle number density has the correct dimension [length $]^{-2}$. It can be readily shown that

$$
\beta P_{N}=n(d)-2 \pi\left(\sigma^{\prime}\right)^{2}
$$

Simultaneously, Eq. (38) implies the exact relation

$$
n(0)-n(d)=2 \pi\left[\sigma^{2}-\left(\sigma^{\prime}\right)^{2}\right]
$$

and we can write

$$
\beta P_{N}=n(0)-2 \pi \sigma^{2} .
$$

Relations (39) and (41) correspond to the contact theorem valid for rectilinear (planar or line) wall surfaces [10,5. It is interesting that the contact theorem has the same form for arbitrary, finite or infinite, circumference $W$.

Our next aim is to perform the continuum of the above formulas in the thermodynamic limit $N, W \rightarrow \infty$, at the fixed ratio $N / W=\sigma+\sigma^{\prime}$. We choose $t=\left(j-W \sigma^{\prime}+\frac{1}{2}\right) / N$ as the continuous variable. For the density profile (38), one obtains

$$
\begin{aligned}
n(x) & =4 \pi\left(\frac{N}{W}\right)^{2} \int_{-\frac{\sigma^{\prime}}{\sigma+\sigma^{\prime}}}^{\frac{\sigma}{\sigma+\sigma^{\prime}}} \mathrm{d} t t \frac{\mathrm{e}^{-4 \pi\left(\sigma+\sigma^{\prime}\right) t x}}{1-\mathrm{e}^{-4 \pi\left(\sigma+\sigma^{\prime}\right) t d}} \\
& =\frac{1}{\pi d^{2}} \int_{-2 \pi \sigma^{\prime} d}^{2 \pi \sigma d} \mathrm{~d} s s \frac{\mathrm{e}^{-2 s x / d}}{1-\mathrm{e}^{-2 s}} .
\end{aligned}
$$

The pressure, given by

$$
\beta P=\frac{1}{2}[n(0)+n(d)]-\pi\left[\sigma^{2}+\left(\sigma^{\prime}\right)^{2}\right],
$$

is found after some algebra in a symmetrized form

$$
\beta P=\frac{1}{2 \pi d^{2}}\left(\int_{0}^{2 \pi \sigma d}+\int_{0}^{2 \pi \sigma^{\prime} d}\right) \mathrm{d} s \frac{s}{\sinh s} \mathrm{e}^{-s} .
$$

In the symmetric case $\sigma=\sigma^{\prime}$, we reproduce the previously obtained formula (4.40) of ref. [25. 


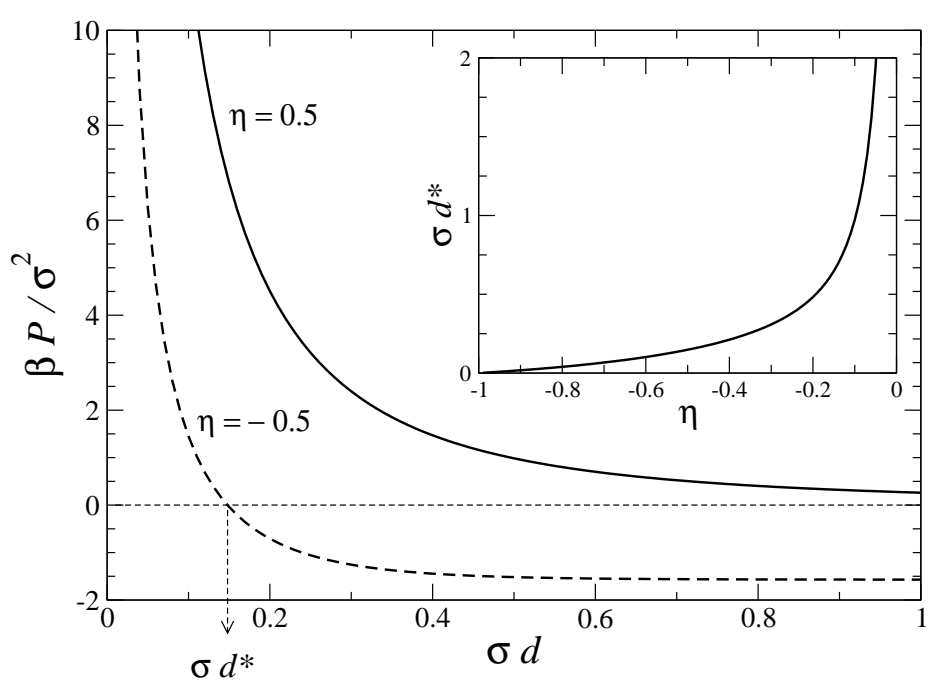

Fig. 3 Unconstrained model: The dimensionless pressure $\beta P / \sigma^{2}$ vs. the dimensionless distance $\sigma d$ for two values of the asymmetry parameter $\eta=1 / 2$ (solid line) and $\eta=-1 / 2$ (dashed line). In both cases, the pressure exhibits a monotonous decrease from $\infty$ at $\sigma d \rightarrow 0$ to: 0 for $\eta=1 / 2$ and the capacitor limit $-2 \pi \eta^{2}$ for $\eta=-1 / 2$ at $\sigma d \rightarrow \infty$. In the latter case, the pressure equals to zero at the equilibrium distance $\sigma d^{*}$ whose dependence on the negative $\eta$ is pictured in the inset.

We want to analyze the dependence of the pressure on the distance $d$ between the charged lines. Using the substitution $s=d t$ in (44), the differentiation with respect to $d$ implies

$$
\frac{\partial}{\partial d} \beta P=-\frac{1}{2 \pi} \int_{-2 \pi \sigma^{\prime}}^{2 \pi \sigma} \mathrm{d} t\left[\frac{t}{\sinh (d t)}\right]^{2}<0,
$$

where the integral is positive with regard to the restriction $-\sigma^{\prime}<\sigma$. This means that $\beta P$ decays monotonously as the function of distance $d$.

In the limit of small distances, we see from (44) that the pressure

$$
\beta P \underset{d \rightarrow 0}{\sim} \frac{\sigma+\sigma^{\prime}}{d}>0
$$

exhibits the singularity and is positive, i.e. there is a repulsion between the charged lines. This small $d$ divergence is expected, and simply stems from the entropy cost of confining the mobile ions in a narrow slab. Quite clearly then, we may anticipate that the constrained model will not exhibit the same $1 / d$ divergence, since approaching the two charged lines does not restrict the configurational space of mobile ions.

The asymptotic large-distance limit of $\beta P$ depends on the sign of $\sigma^{\prime}$.

- If $\sigma^{\prime}>0$ (like-charged lines), we have the universal result

$$
\beta P \underset{d \rightarrow \infty}{\sim} \frac{1}{\pi d^{2}} \int_{0}^{\infty} \mathrm{d} s \frac{s}{\sinh s} \mathrm{e}^{-s}=\frac{\pi}{12} \frac{1}{d^{2}},
$$


independent of the amplitudes of $\sigma$ and $\sigma^{\prime}$ 1 . It coincides with the previous finding (8) for symmetrically charged lines; the same phenomenon is observed in the mean-field limit where the formula (7) takes place for any $\sigma^{\prime}>0$ [14]. The pressure goes to zero at $d \rightarrow \infty$ from above, in agreement with its monotonous decrease property from $+\infty$ at $d \rightarrow 0$. Another consequence is that the pressure is positive (repulsive) for every $d$; the value of the coupling $\Gamma=2$ is not large enough to obtain attraction between the like-charged lines. The plot of the dimensionless pressure $\beta P / \sigma^{2}$ vs. the dimensionless distance $\sigma d$ for $\eta=1 / 2$ is pictured in Fig. 3 by the solid line. The regions close to the two charged lines decouple from one another in the limit $d \rightarrow \infty$, the densities of particles at the lines $n(0)=2 \pi \sigma^{2}$ and $n(d)=2 \pi\left(\sigma^{\prime}\right)^{2}$ being equivalent to those for semi-infinite geometries.

- For a neutral line at $x=d, \sigma^{\prime}=0$, we have

$$
\beta P \underset{d \rightarrow \infty}{\sim} \frac{1}{2 \pi d^{2}} \int_{0}^{\infty} \mathrm{d} s \frac{s}{\sinh s} \mathrm{e}^{-s}=\frac{\pi}{24} \frac{1}{d^{2}} .
$$

As before, the pressure is always positive. Comparing this result with (47) we see that the neutrality of the line at $x=d$ diminishes the asymptotic pressure by $1 / 2$. In the mean-field limit, the diminishing factor is equal to $1 / 4$ [14]; that factor $1 / 4$ is obtained by redefining $d \rightarrow 2 d$ which was argued to be related to the decomposition of a system with an asymmetry parameter $\eta>0$ into two halves each with an effective $\eta=0$. Such an argument, which discards fluctuations, no longer applies to the coupling constant $\Gamma=2$.

- If $\sigma^{\prime}<0$ (oppositely charged lines), we reexpress the second integral on the rhs of (44) by using the substitution $s \rightarrow-s$ and afterwards writing $\mathrm{e}^{s}=\mathrm{e}^{s}-\mathrm{e}^{-s}+\mathrm{e}^{-s}$, with the result

$$
-\left(2 \pi \sigma^{\prime} d\right)^{2}-\int_{0}^{-2 \pi \sigma^{\prime} d} \mathrm{~d} s \frac{s}{\sinh s} \mathrm{e}^{-s} .
$$

Thus, at large distances, the pressure does not vanish,

$$
\beta P \underset{d \rightarrow \infty}{\sim}=-2 \pi\left(\sigma^{\prime}\right)^{2}
$$

with an exponentially decaying correction. This behaviour is not surprising. Since the sign of the particle charge is the same as that of the line at $x=d$, particles cannot neutralize and are repelled from this line. For sufficiently distant lines we have $n(0) \simeq 2 \pi\left[\sigma^{2}-\left(\sigma^{\prime}\right)^{2}\right]$ and $n(d) \simeq 0$, i.e. all counter-ions stay in the neighbourhood of line at $x=0$ and compensate partially its line charge density $\sigma e$ to $\left|\sigma^{\prime}\right| e$. We are left with a capacitor of two lines with opposite charge densities $\pm \sigma^{\prime} e$ for which the pressure is negative (attractive) and given just by Eq. (49). The same scenario was discussed in 21, and occurs in the mean-field treatment [14. The dependence of $\beta P / \sigma^{2}$ on $\sigma d$ for $\eta=-1 / 2$ is represented in Fig. 3 by the

\footnotetext{
1 Note that whenever the pressure is of the form $\beta P \propto 1 / d^{2}$, it cannot depend on the absolute values of $\sigma$ and $\sigma^{\prime}$, but only on the ratio $\eta=\sigma / \sigma^{\prime}$, for dimensional reasons. In the symmetric case, it is thus $\sigma$ independent.
} 
dashed line. Since $\beta P$ decays monotonously from $+\infty$ at $d \rightarrow 0$ to the negative number $-2 \pi\left(\sigma^{\prime}\right)^{2}$ at $d \rightarrow \infty$, there exists just one equilibrium distance $d^{*}$ at which $\beta P\left(d^{*}\right)=0$. Thus, oppositely charged lines repel one another for distances $d<d^{*}$ while there is an attraction for $d>d^{*}$. The dependence of $\sigma d^{*}$ on the negative asymmetry $\eta$ is presented in the inset of Fig. 3.

\section{Counter-ions at charged walls}

The constrained model in Fig. 2 possesses a non-trivial $\epsilon$-function (22). At $\Gamma=2$, the renormalized one-body weight (25) reads

$$
w_{\text {ren }}(x)=\frac{4 \pi \lambda}{W^{2}}[\delta(x)+\delta(x-d)] \exp \left[2 \pi\left(2 \sigma^{\prime}-\frac{1}{W}\right) x\right]
$$

and the diagonal interaction elements (28) are given by

$$
w_{j}=\frac{4 \pi \lambda}{W}\left[1+\mathrm{e}^{-\frac{4 \pi d}{W}\left(j-W \sigma^{\prime}+\frac{1}{2}\right)}\right]
$$

$(j=0,1, \ldots, N-1)$. The pressure (33) is given by

$$
\begin{aligned}
\beta P_{N}= & -2 \pi\left(\sigma^{\prime}\right)^{2}-\frac{4 \pi}{W^{2}} \sum_{j=0}^{N-1} \frac{j-W \sigma^{\prime}+\frac{1}{2}}{1+\mathrm{e}^{-\frac{4 \pi d}{W}\left(j-W \sigma^{\prime}+\frac{1}{2}\right)}} \\
& \times \mathrm{e}^{-\frac{4 \pi d}{W}\left(j-W \sigma^{\prime}+\frac{1}{2}\right)},
\end{aligned}
$$

which is of course independent of $\lambda$. It is easy to show that this relation is equivalent to the following one

$$
\beta P_{N}=-2 \pi \sigma^{2}+\frac{4 \pi}{W^{2}} \sum_{j=0}^{N-1} \frac{j-W \sigma^{\prime}+\frac{1}{2}}{1+\mathrm{e}^{-\frac{4 \pi d}{W}\left(j-W \sigma^{\prime}+\frac{1}{2}\right)}},
$$

so that we can write

$$
\begin{aligned}
\beta P_{N}= & -\pi\left[\sigma^{2}+\left(\sigma^{\prime}\right)^{2}\right]+\frac{2 \pi}{W^{2}} \sum_{j=0}^{N-1}\left(j-W \sigma^{\prime}+\frac{1}{2}\right) \\
& \times \tanh \left[\frac{2 \pi d}{W}\left(j-W \sigma^{\prime}+\frac{1}{2}\right)\right] .
\end{aligned}
$$

The continuum procedure, analogous to that for the unconstrained model, leads to

$$
\beta P=-\frac{1}{2 \pi d^{2}}\left(\int_{0}^{2 \pi \sigma d}+\int_{0}^{2 \pi \sigma^{\prime} d}\right) \mathrm{d} s \frac{s}{\cosh s} \mathrm{e}^{-s} .
$$

The density profile (34) has the form

$$
n(x)=n_{0} \delta(x)+n_{d} \delta(x-d),
$$


where

$$
n_{0}=\frac{1}{W} \sum_{j=0}^{N-1} \frac{1}{1+\mathrm{e}^{-\frac{4 \pi d}{W}\left(j-W \sigma^{\prime}+\frac{1}{2}\right)}}
$$

and

$$
n_{d}=\frac{1}{W} \sum_{j=0}^{N-1} \frac{1}{1+\mathrm{e}^{-\frac{4 \pi d}{W}\left(j-W \sigma^{\prime}+\frac{1}{2}\right)}} \mathrm{e}^{-\frac{4 \pi d}{W}\left(j-W \sigma^{\prime}+\frac{1}{2}\right)} .
$$

The prefactors $n_{0}$ and $n_{d}$ to the Dirac delta functions are the line particle densities of dimension [length $]^{-1}$ along the $y$-axis at $x=0$ and $x=d$, respectively. They are constrained by the obvious electroneutrality condition

$$
n_{0}+n_{d}=\frac{N}{W}=\sigma+\sigma^{\prime} .
$$

After taking the continuum limits of (57) and (58), we get

$$
\begin{aligned}
& n_{0}=\sigma+\frac{1}{4 \pi d} \ln \left(\frac{1+\mathrm{e}^{-4 \pi \sigma d}}{1+\mathrm{e}^{-4 \pi \sigma^{\prime} d}}\right), \\
& n_{d}=\sigma^{\prime}+\frac{1}{4 \pi d} \ln \left(\frac{1+\mathrm{e}^{-4 \pi \sigma^{\prime} d}}{1+\mathrm{e}^{-4 \pi \sigma d}}\right) .
\end{aligned}
$$

It holds that $0 \leq n_{d} \leq n_{0}$. Note that for any finite distance $d$, (and symmetric $\sigma=\sigma^{\prime}$ case excluded), the net (fixed background plus mobile particle) charge density on each of the two lines is nonzero: $\left(\sigma-n_{0}\right) e \neq 0$ and $\left(\sigma^{\prime}-n_{d}\right) e=$ $-\left(\sigma-n_{0}\right) e \neq 0$. The neutrality of the two lines is attained at asymptotically large $d$ provided that $\sigma^{\prime} \geq 0$. If $\sigma^{\prime}<0$, we find that $n_{0}=\sigma+\sigma^{\prime}$ and $n_{d}=0$, i.e. the system corresponds to a capacitor of two lines with opposite charge densities $\pm \sigma^{\prime} e$. The ratio $n_{d} / n_{0}$ as a function of the dimensionless distance $\sigma d$ is drawn for two values of the asymmetry parameter $\eta=1 / 2$ (solid line) and $\eta=-1 / 2$ (dashed line) in Fig. 4. It is seen that $n_{d} / n_{0} \rightarrow 1$ for $\sigma d \rightarrow 0$ for every positive or negative value of $\eta$. This is an entropy driven phenomenon, since a particle's localization on one or the other line is at small $d$ inconsequential from the energetic point of view. For $\eta=1 / 2$, the ratio $n_{d} / n_{0}$ tends to the neutrality value $1 / 2$ at $\sigma d \rightarrow \infty$. On the other hand, for $\eta=-1 / 2$, the ratio $n_{d} / n_{0}=0$ at $\sigma d \rightarrow \infty$, in agreement with the above discussion. It is interesting to note that for negative $\eta$ the value of $n_{d}$ is strictly positive for finite values of $\sigma d$ : although the energy is increased by putting particles to the likely charged line, the entropy increase on this less populated line favors a non-vanishing occupation by particles.

It can be shown from the expression of the pressure (55) that

$$
\frac{\partial}{\partial d} \beta P=\frac{1}{2 \pi} \int_{-2 \pi \sigma^{\prime}}^{2 \pi \sigma} \mathrm{d} t\left[\frac{t}{\cosh (d t)}\right]^{2}>0,
$$

i.e. the pressure is a monotonously increasing function of $d$. In the limit $d \rightarrow 0, \beta P$ is negative:

$$
\beta P \underset{d \rightarrow 0}{\sim}=-\pi\left[\sigma^{2}+\left(\sigma^{\prime}\right)^{2}\right]
$$




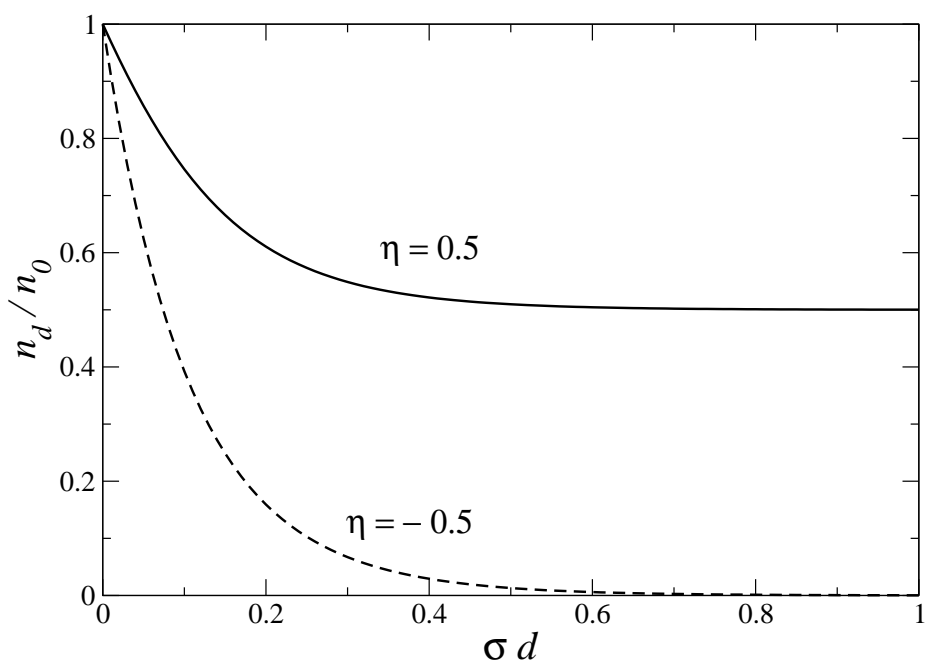

Fig. 4 Constrained model: The ratio of the line particle densities $n_{d} / n_{0}$ vs. distance $\sigma d$ for the asymmetry $\eta=1 / 2$ (solid line) and $\eta=-1 / 2$ (dashed line). In both cases, the ratio decreases monotonously from 1 at $\sigma d \rightarrow 0$ to: the electroneutrality limit 0.5 for $\eta=1 / 2$ and 0 for $\eta=-1 / 2$ at $\sigma d \rightarrow \infty$.

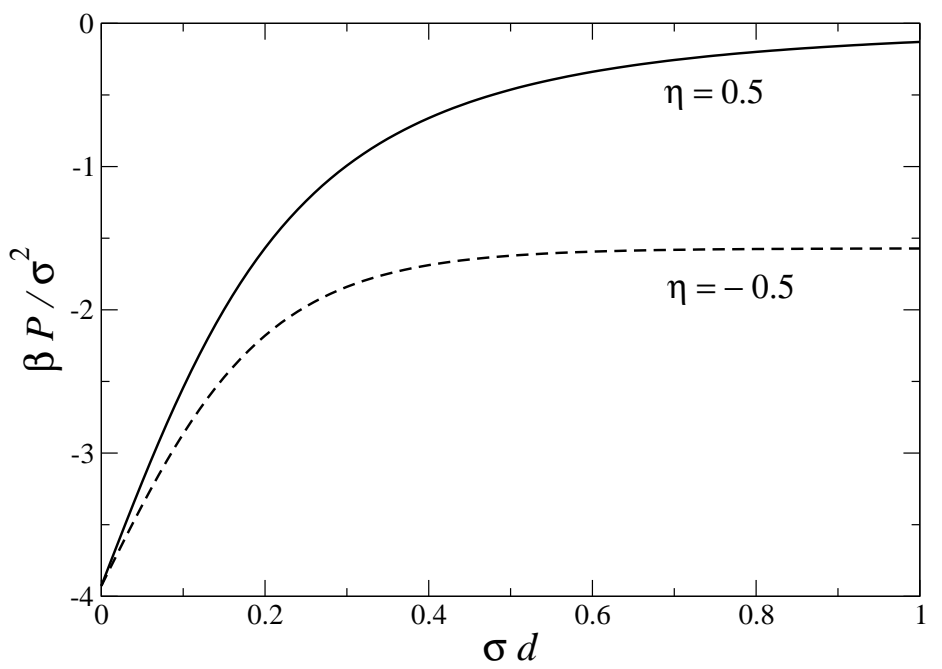

Fig. 5 Constrained model: Dimensionless pressure $\beta P / \sigma^{2}$ as a function of dimensionless distance $\sigma d$ for the asymmetry $\eta=1 / 2$ (solid line) and $\eta=-1 / 2$ (dashed line). In both cases, the pressure exhibits a monotonous increase from $-\pi\left(1+\eta^{2}\right)$ at $\sigma d \rightarrow 0$ to: 0 for $\eta=1 / 2$ and the capacitor limit $-2 \pi \eta^{2}$ for $\eta=-1 / 2$ at $\sigma d \rightarrow \infty$. 
and, as expected, does not exhibit any singularity.

At asymptotically large distances, the sign of $\sigma^{\prime}$ is important.

- If $\sigma^{\prime}>0$, one obtains the universal formula

$$
\beta P \underset{d \rightarrow \infty}{\sim}-\frac{1}{\pi d^{2}} \int_{0}^{\infty} \mathrm{d} s \frac{s}{\cosh s} \mathrm{e}^{-s}=-\frac{\pi}{24} \frac{1}{d^{2}} .
$$

This asymptotic behaviour coincides with that of the van der WaalsCasimir for two neutral 2D conductors (44); since $n_{0}=\sigma$ and $n_{d}=\sigma^{\prime}$ at infinite distance, each of the two lines is indeed neutral and the force between the lines results from internal charge fluctuations inside each of the lines. The pressure goes to zero at $d \rightarrow \infty$ from below, which agrees with its property of the monotonous increase from the negative value (63) at $d \rightarrow 0$. The pressure is always negative (attractive) for the couple of like-charged lines. The dimensionless pressure plot for $\eta=1 / 2$ is presented in Fig. 5 by the solid line.

- For $\sigma^{\prime}=0$, we find that

$$
\beta P \underset{d \rightarrow \infty}{\sim}-\frac{1}{2 \pi d^{2}} \int_{0}^{\infty} \mathrm{d} s \frac{s}{\sinh s} \mathrm{e}^{-s}=-\frac{\pi}{48} \frac{1}{d^{2}},
$$

which is one half of the previous result (64). As before, the pressure is negative.

- If $\sigma^{\prime}<0$, we reexpress the second integral on the rhs of (55) as

$$
\left(2 \pi \sigma^{\prime} d\right)^{2}-\int_{0}^{-2 \pi \sigma^{\prime} d} \mathrm{~d} s \frac{s}{\cosh s} \mathrm{e}^{-s}
$$

and arrive at the capacitor result

$$
\beta P \underset{d \rightarrow \infty}{\sim}=-2 \pi\left(\sigma^{\prime}\right)^{2} .
$$

Since the monotonously increasing $\beta P$ interpolates between two negative values as $d$ goes from 0 to $\infty$, the pressure is always negative. For the asymmetry parameter $\eta=-1 / 2, \beta P / \sigma^{2}$ as the function of $\sigma d$ is pictured in Fig. 15 by the dashed line.

\section{Conclusion}

In this paper, we investigated thermal equilibrium of 2D Coulomb systems composed of two parallel asymmetrically charged lines at distance $d$, neutralized by "counter-ions only". Two versions of the model were considered: in the unconstrained version, counter-ions move freely in the region between the lines, while in the constrained version, counter-ions stick to the charged lines. For the exactly solvable coupling constant $\Gamma=2$, we analyzed the dependence of the pressure on $d$, especially its sign and the asymptotic large- $d$ behaviour.

The two models exhibit some common features. The expressions for the pressure (44) and (55) consist of the sum of two decoupled contributions, one 
from each line. Such property does not occur in the weak-coupling limit and is probably related to the free-fermion nature of the $\Gamma=2$ coupling. According to the derivative formulas (45) and (62), the pressure is always a monotonous function of $d$, decreasing for the unconstrained model, and increasing for its constrained counterpart. A similarly increasing pressure is found for bilayers, frozen in their ground state (with thus a diverging coupling constant) [26].

For likely charged lines, the pressure goes to 0 at large $d$ without changing its sign: it is always positive (repulsive) for the unconstrained model and negative (attractive) for the constrained model. The large-distance asymptotic behaviour of the pressure for the unconstrained model (47) is universal, however, the prefactor to $1 / d^{2}$ is not consistent with the mean-field prediction (7) but has to be renormalized by a temperature-dependent function. This remark on the failure of mean-field at asymptotic distances, corroborates previous $2 \mathrm{D}$ results obtained for symmetric lines 25 . It goes against common expectation that mean-field should hold at large $d$ 27,28, the underlying argument being that the small density of ions far from the plate may effectively drive the system into a weakly-coupled regime, in the corresponding distance range. However, it should be kept in mind that Coulomb potential $v(\mathbf{r})$ is logarithmic in two dimensions and hence scale-free, so that the Coulombic coupling does not depend on the density: it is always $\beta e^{2}$. Hence, a 2D system, unlike its higher dimensional counterparts, is nowhere weakly coupled when $\Gamma>1$, and it therefore does not come as a surprise that mean-field breaks down in 2D, even at large distances. Conversely, in dimension $\nu=3$, the standard argument on the asymptotic validity of meanfield may well apply. We note that it is backed up by recent accurate Monte Carlo results 29. As concerns the constrained model, the net charge density on each of the two lines is nonzero for every finite distance $d$ and vanishes only at $d \rightarrow \infty$. The universal $d \rightarrow \infty$ behavior of the pressure (64) coincides with that of the van der Waals-Casimir for two neutral 2D conductors (4), without any need for temperature renormalization.

For oppositely charged lines, the pressure at asymptotically large $d$ corresponds to a capacitor in both unconstrained (49) and constrained (66) cases. This fact has an important impact especially on the unconstrained model which exhibits at a special dimensionless distance $\sigma d^{*}$ a transition from the repulsion regime for $d<d^{*}$ to the attraction regime for $d>d^{*}$; for the dependence of $\sigma d^{*}$ on the negative asymmetry $\eta \equiv \sigma^{\prime} / \sigma$ see the inset of Fig. 3.

Finally, investigating the two present models under stronger correlations (e.g. $\Gamma=4$, or 6 ) and also ultimately in the strongly coupled limit $(\Gamma \rightarrow \infty)$, provides interesting venues for future work.

Acknowledgements The support received from Grant VEGA No. 2/0049/12 is acknowledged.

\section{References}

1. Andelman, D.: Introduction to electrostatics in soft and biological matter. In Soft Condensed Matter Physics in Molecular and Cell Biology, ed. W.C.K. 
Poon, D. Andelman, Chapt. 6. Taylor \& Francis, New York (2006)

2. Bordag, M., Mohideen, U., Mostepanenko, V.M.: New developments in the Casimir effect. Phys. Rep. 353, 1-205 (2001)

3. Buenzli, P.R., Martin, Ph.A.: Microscopic origin of universality in Casimir forces. J. Stat. Phys. 119, 273-307 (2005)

4. Burak, Y., Andelman, D.: Test-charge theory for the electric double layer. Phys. Rev. E 70, $016102(2004)$

5. Carnie, S.L., Chan, D.Y.C.: The statistical mechanics of the electrical double layer: Stress tensor and contact conditions. J. Chem. Phys. 74, 1293-1297 (1981)

6. Choquard, Ph.: The two-dimensional one component plasma on a periodic strip. Helv. Phys. Acta 54, 332-332 (1981)

7. Cornu, F., Jancovici, B.: On the two-dimensional Coulomb gas. J. Stat. Phys. 49, 33-56 (1987)

8. Cornu, F., Jancovici, B.: The electrical double layer: A solvable model. J. Chem. Phys. 90, 2444-2452 (1989)

9. Forrester, P.J.: Exact results for two-dimensional Coulomb systems. Phys. Rep. 301, 235-270 (1998)

10. Henderson, D., Blum, L.: Some exact results and the application of the mean spherical approximation to charged hard spheres near a charged hard wall. J. Chem. Phys. 69, 5441-5449 (1978)

11. Jancovici, B.: Exact results for the two-dimensional one-component plasma. Phys. Rev. Lett. 46, 386-388 (1981)

12. Jancovici, B.: Inhomogeneous two-dimensional plasmas. In Inhomogeneous Fluids, ed. D. Henderson, pp. 201-237. Dekker, New York (1992)

13. Jancovici, B., Šamaj, L.: Screening of classical Casimir forces by electrolytes in semi-infinite geometries. J. Stat. Mech., P08006 (2004)

14. Kanduč, M., Trulsson, M., Naji, A., Burak, Y., Forsman, J., Podgornik, R.: Weak- and strong-coupling electrostatic interactions between asymmetrically charged planar surfaces. Phys. Rev. E 78, 061105 (2008)

15. Lau, A.W.C., Levine, D., Pincus, P.: Novel electrostatic attraction from plasmon fluctuations. Phys. Rev. Lett. 84, 4116-4119 (2000)

16. Lau, A.W.C., Pincus, P., Levine, D., Fertig, H.A.: Electrostatic attraction of coupled Wigner crystals: Finite temperature effects. Phys. Rev. E 63, 051604 (2001)

17. Martin, Ph.A.: Sum rules in charged fluids. Rev. Mod. Phys. 60, 1075-1127 (1988)

18. Milton, K.A.: The Casimir effect. World Scientific, London (2001)

19. Netz, R.R., Orland, H.: Beyond Poisson-Boltzmann: Fluctuation effects and correlation functions. Eur. Phys. J. E 1, 203-214 (2000)

20. Netz, R.R.: Electrostatics of counter-ions at and between planar charged walls: From Poisson-Boltzmann to the strong-coupling theory. Eur. Phys. J. E 5, 557-574 (2001)

21. Paillusson, F., Trizac, E.: Interaction regimes for oppositely charged plates with multivalent counterions. Phys. Rev. E 84, 011407 (2011)

22. Šamaj, L., Percus, J.K.: A functional relation among the pair correlations of the two-dimensional one-component plasma. J. Stat. Phys. 80, 811-824 (1995)

23. Šamaj, L., Wagner, J., Kalinay, P.: Translation symmetry breaking in the onecomponent plasma on the cylinder. J. Stat. Phys. 117, 159-178 (2004)

24. Šamaj, L., Trizac, E.: Counterions at highly charged interfaces: From one plate to like-charge attraction. Phys. Rev. Lett. 106, 078301 (2011)

25. Šamaj, L., Trizac, E.: Counter-ions at charged walls: Two-dimensional systems. Eur. Phys. J. E 34, 20 (2011)

26. Šamaj, L., Trizac, E.: Critical phenomena and phase sequence in a classical bilayer Wigner crystal at zero temperature. Phys. Rev. B 85, 205131 (2012)

27. Shklovskii, B.I.: Screening of a macroion by multivalent ions: Correlationinduced inversion of charge. Phys. Rev. E 60, 5802-5811 (1999)

28. dos Santos, A.P., Diehl, A., Levin, Y.: Electrostatic correlations in colloidal suspensions: density profiles and effective charges beyond the Poisson-Boltzmann theory. J. Chem. Phys. 130, 124110 (2009) 
29. J.P. Mallarino, G. Téllez, E. Trizac, Counter-ion density profile around charged cylinders: the strong-coupling needle limit. J. Phys. Chem. B 117, 12702 (2013) 